\title{
Use of Preoperative Radiation Therapy in Early- stage and Locally Advanced Breast Cancer
}

Julie L. Koenig ${ }^{1}$, Margaret M. Kozak ${ }^{2}$, Aaron Sabolch ${ }^{3}$, Kathleen Horst ${ }^{4}$, Jillian Tsai ${ }^{5}$, Irene L. Wapnir ${ }^{6}$, Erqi Pollom 4

1. School of Medicine, Stanford University, Stanford, USA 2. Radiation Oncology, Stanford Cancer Institute, Stanford, USA 3. Radiation Oncology, Kaiser Permanente Interstate Radiation Oncology Center, Portland, USA 4. Radiation Oncology, Stanford University, Stanford, USA 5. Radiation Oncology, Memorial Sloan Kettering Cancer Center, New York, USA 6. Surgery, Stanford Cancer Institute, Stanford, USA

Corresponding author: Julie L. Koenig, jlkoenig12@gmail.com

\section{Abstract}

\section{Purpose}

There is growing interest in delivering radiation preoperatively (preopRT) rather than postoperatively (postopRT) for breast cancer. Using the National Cancer Database, we evaluated the use and outcomes of preopRT in breast cancer.

\section{Methods}

We identified adult females diagnosed with non-metastatic breast cancer treated with definitive surgery and radiation between 2004 and 2014. Logistic regression models evaluated factors associated with use of preopRT in early-stage (clinical T1-3/N0-1) and locally advanced (clinical T4/N2-3) disease. Rates of breastconserving surgery, breast reconstruction, positive surgical margins, and 30-day surgical readmissions were compared between patients receiving preopRT and postopRT.

\section{Results}

Of 373,595 patients who met our inclusion criteria, 1,245 (0.3\%) patients received preopRT. Patients receiving preopRT were more likely to be of lower socioeconomic status and have tumors with higher $\mathrm{T}$ stage. Younger age and N1 (vs N0) disease predicted for use of preopRT in early-stage disease, while older age and NO disease predicted for use of preopRT in the locally advanced setting. PreopRT patients were less likely to undergo breast-conserving surgery and more likely to have positive surgical margins. Rates of unplanned readmissions within 30 days of surgery were similar among patients treated with preopRT and postopRT.

\section{Conclusions}

Received 04/28/2019

Review began 08/07/2019 Review ended 09/20/2019 Published 09/24/2019

\section{(c) Copyright 2019}

Koenig et al. This is an open access article distributed under the terms of the Creative Commons Attribution License CC-BY 3.0., which permits unrestricted use, distribution, and reproduction in any medium, provided the original author and source are credited.
PreopRT is a new treatment strategy for patients with breast cancer with different clinical and sociodemographic drivers of its use in the early-stage and locally advanced settings. We await the results of clinical trials studying the efficacy of this approach.

Categories: Radiation Oncology, General Surgery, Oncology

Keywords: preoperative radiation therapy, neoadjuvant treatment, breast cancer, national cancer database

\section{Introduction}

Many patients with breast cancer are treated with a combination of surgery, chemotherapy, and radiation therapy (RT). Historically, RT has been delivered following surgery and chemotherapy, but there has been growing interest in delivering RT preoperatively. Preoperative RT (preopRT) can potentially downstage tumors, more accurately target tumor, thereby reducing soft tissue toxicity due to smaller treatment volumes [1], improve rates of pathologic complete response (pCR), and facilitate margin-negative resection [2]. Another emerging concept is that of RT as an effective tumor vaccine when directed to intact tumor, activating a robust antitumor immune response and eradicating subclinical disease [3, 4].

While preopRT has been extensively investigated for inoperable and locally advanced breast cancers, there has been less data on the use and efficacy of preopRT for early-stage (T1-3/N0-1) disease. Several recently reported phase I and II trials evaluating the use of preopRT for early-stage breast cancer have shown that this approach is feasible and well-tolerated [1] with good to excellent cosmetic outcomes [5, 6] and local control rates upwards of $90 \%[6,7]$.

Using a large hospital-based national cancer registry, we sought to assess factors associated with receipt of preopRT for early-stage and locally advanced breast cancer, and evaluate the surgical management and 


\section{Materials And Methods \\ Data source}

We performed a retrospective cohort study using the National Cancer Database (NCDB) 2014 Participant User File. NCDB is a joint program of the Commission on Cancer (CoC) of the American College of Surgeons and the American Cancer Society (ACS) and is a hospital-based registry with data from more than 1,500 CoCaccredited hospitals. It includes information about demographics, disease stage, and first course of treatment for $70 \%$ of newly diagnosed cancer cases in the United States. The $\mathrm{CoC}$ and American Cancer Society have not verified and are not responsible for the analytic or statistical methodology used or for the conclusions drawn from these data. This study was exempt from review by our institutional review board.

\section{Patient selection}

We identified adult women who were diagnosed with non-metastatic invasive breast cancer between 2004 and 2014. We restricted our study to patients who underwent definitive surgery and preoperative or postoperative external beam RT. Preoperative RT (preopRT) was defined as RT that started within the year prior to definitive surgery based on variables for time of RT and definitive surgery relative to date of diagnosis. Postoperative RT (postopRT) was defined as radiation delivered within six months of surgery. Radiation dose was limited to 14 to $70 \mathrm{~Gy}$ based on studies including treatment doses within this range and a small expansion of the range to account for variability in coding [1, 8]. We excluded patients whose RT course extended longer than 10 weeks. Additional inclusion and exclusion criteria are listed in Table 1.

\section{Covariates}

Early-stage disease was defined as clinically T1-3/N0-1 and locally advanced was defined as clinically T4/N23. Chemotherapy and hormone therapy initiated within one year before definitive surgery were considered neoadjuvant. Adjuvant systemic therapies were similarly defined as therapies initiated within a year after surgery. The NCDB variable for human epidermal growth factor receptor 2 (HER2) was based on information from immunohistochemistry and fluorescence in situ hybridization (FISH) and was consistently available starting in 2010. Results from FISH took precedence when both tests were available. Outcome variables included pathologic complete response (pCR), surgical modality (breast-conserving surgery vs mastectomy), breast reconstruction, surgical margins, and 30-day surgical readmission. NCDB codes pCR based on review of the medical record for a physician statement about response to neoadjuvant therapy. Thirty-day surgical readmission was coded based on an unplanned readmission within 30 days of discharge following hospitalization for resection of the primary cancer.

\section{Statistical analyses}

Demographic, tumor, treatment characteristics, and outcomes were compared between patients who received preopRT and postopRT using Chi-square, Fisher's exact, and Wilcoxon rank sum tests.

Univariate and multivariate logistic regression models were used to determine factors associated with use of preopRT. Our models were adjusted for demographic and tumor factors that were selected a priori. Given that treatment facility type was not available for patients under 40 years old and complete molecular subtype information was not available until after 2010, we constructed separate models additionally adjusting for these factors.

All tests were two-sided with an alpha value of 0.05. Statistical analyses were performed using STATA/SE software (version 14.0, StataCorp, College Station, TX).

\section{Results}

\section{Factors associated with receipt of preoperative RT}

We identified 373,595 patients who met our inclusion criteria (Table 1). A total of 1,245 (0.3\%) patients received preopRT; 850 (68\%) of these patients had early-stage disease (clinical T1-3/N0-1) and 395 (32\%) had locally advanced disease (clinical T4/N2-3). Table 2 shows baseline characteristics of patients who received preopRT versus postopRT. In general, patients who received preopRT were more likely to be black, uninsured/Medicaid-insured, living in zip codes of lower median income and educational attainment, and with higher grade and more locally advanced tumors. 


\section{Cureus}

Cohort Selection

1 Total breast cancer cases diagnosed from 2004-2014

Limit to female patients 18 years and older with histologically confirmed non-metastatic, primary invasive breast cancer

3 Limit to patients who underwent definitive surgery

Limit to patients who underwent preoperative or postoperative external beam radiation therapy within one year and six months of surgery, respectively

5 Exclude patients with a prior cancer diagnosis

$6 \quad$ Exclude patients with incomplete clinical staging

7 Exclude patients with incomplete radiation and surgical treatment timing

8 Limit radiation dose to 14 to 70 Gy

9 Exclude patients whose radiation therapy course extended longer than 10 weeks

10 Exclude patients whose diagnosis date precedes reference date to ensure data completeness

11 Exclude patients diagnosed at autopsy
No.

$2,246,280$

$100.00 \%$

$1,549,168 \quad 68.97 \%$

$1,491,650 \quad 66.41 \%$

$673,899 \quad 30.00 \%$

$599,042 \quad 26.67 \%$

$439,705 \quad 19.57 \%$

$428,704 \quad 19.09 \%$

$388,171 \quad 17.28 \%$

$378,703 \quad 16.86 \%$

$373,596 \quad 16.63 \%$

$373,595 \quad 16.63 \%$

\section{TABLE 1: Cohort selection.}

\begin{tabular}{|c|c|c|c|}
\hline & PostopRT & PreopRT & \\
\hline Characteristics & No. (\%) & No. (\%) & $\mathrm{p}$ \\
\hline Total & $372,350(99.7 \%)$ & $1245(0.3 \%)$ & \\
\hline Age (years) & & & $<0.001$ \\
\hline $18-50$ & $91,839(24.7 \%)$ & 393 (31.6\%) & \\
\hline $51-64$ & 145,778 (39.2\%) & $500(40.2 \%)$ & \\
\hline$\geq 65$ & 134,733 (36.2\%) & $352(28.3 \%)$ & \\
\hline Age (years), median (IQR) & $60(51,68)$ & $57(48,65)$ & $<0.001$ \\
\hline Race & & & $<0.001$ \\
\hline White & $315,874(84.8 \%)$ & 959 (77.0\%) & \\
\hline Black & $38,205(10.3 \%)$ & 213 (17.1\%) & \\
\hline Other/Unknown & $18,271(4.9 \%)$ & 73 (5.9\%) & \\
\hline Year of diagnosis & & & $<0.001$ \\
\hline 2004-2007 & 63,488 (17.1\%) & $305(24.5 \%)$ & \\
\hline 2008-2009 & 71,467 (19.2\%) & $232(18.6 \%)$ & \\
\hline 2010-2012 & $134,679(36.2 \%)$ & 447 (35.9\%) & \\
\hline 2013-2014 & $102,716(27.6 \%)$ & $261(21.0 \%)$ & \\
\hline Charlson-Deyo Score & & & 0.004 \\
\hline 0 & $320,714(86.1 \%)$ & 1108 (89.0\%) & \\
\hline$\geq 1$ & $51,636(13.9 \%)$ & $137(11.0 \%)$ & \\
\hline Insurance Coverage & & & $<0.001$ \\
\hline Uninsured & 6953 (1.9\%) & 46 (3.7\%) & \\
\hline
\end{tabular}




\section{Cureus}

\begin{tabular}{|c|c|c|c|}
\hline Private/Managed Care & $214,276(57.5 \%)$ & $665(53.4 \%)$ & \\
\hline Medicare & $122,365(32.9 \%)$ & $349(28.0 \%)$ & \\
\hline Medicaid & $21,337(5.7 \%)$ & $147(11.8 \%)$ & \\
\hline Unknown/Other & $7419(2.0 \%)$ & $38(3.1 \%)$ & \\
\hline Facility type $^{a}$ & & & $<0.001$ \\
\hline Academic/research ${ }^{\mathrm{b}}$ & $103,244(27.7 \%)$ & $395(31.7 \%)$ & \\
\hline Non-academic & $251,782(67.6 \%)$ & 745 (59.8\%) & \\
\hline Unknown & $17,324(4.7 \%)$ & 105 (8.4\%) & \\
\hline Location $^{\mathrm{a}}$ & & & $<0.001$ \\
\hline Northeast & $81,240(21.8 \%)$ & $223(17.9 \%)$ & \\
\hline South & $110,072(29.6 \%)$ & $434(34.9 \%)$ & \\
\hline Central & $100,713(27.0 \%)$ & $290(23.3 \%)$ & \\
\hline West & 63,001 (16.9\%) & $193(15.5 \%)$ & \\
\hline Unknown & $17,324(4.7 \%)$ & 105 (8.4\%) & \\
\hline Income $e^{c}$ & & & $<0.001$ \\
\hline$<\$ 38,000$ & 49,701 (13.3\%) & $221(17.8 \%)$ & \\
\hline$\geq \$ 38,000$ & $320,551(86.1 \%)$ & $1012(81.3 \%)$ & \\
\hline Unknown & 2098 (0.6\%) & $12(1.0 \%)$ & \\
\hline$\%$ without $\mathrm{HSD}^{\mathrm{C}}$ & & & $<0.001$ \\
\hline$\geq 13 \%$ & $131,096(35.2 \%)$ & $519(41.7 \%)$ & \\
\hline$<13 \%$ & $239,281(64.3 \%)$ & 714 (57.3\%) & \\
\hline Unknown & $1973(0.5 \%)$ & $12(1.0 \%)$ & \\
\hline Distance from facility & & & $<0.001$ \\
\hline$\leq 50 \mathrm{mi}$ & 351,235 (94.3\%) & $1132(90.9 \%)$ & \\
\hline$>50 \mathrm{mi}$ & $19,189(5.2 \%)$ & 101 (8.1\%) & \\
\hline Unknown & $1926(0.5 \%)$ & $12(1.0 \%)$ & \\
\hline Residence Type & & & 0.085 \\
\hline Metro & 313,184 (84.1\%) & 1036 (83.2\%) & \\
\hline Urban/rural & 48,943 (13.1\%) & $162(13.0 \%)$ & \\
\hline Unknown & $10,223(2.7 \%)$ & $47(3.8 \%)$ & \\
\hline Tumor Size (cm), median (IQR) & $1.5(1.0,2.5)$ & $2.5(1.3,5.0)$ & $<0.001$ \\
\hline AJCC Clinical T Stage & & & $<0.001$ \\
\hline 1 & $252,738(67.9 \%)$ & $487(39.1 \%)$ & \\
\hline 2 & $87,504(23.5 \%)$ & 301 (24.2\%) & \\
\hline 3 & $21,228(5.7 \%)$ & $152(12.2 \%)$ & \\
\hline 4 & $10,880(2.9 \%)$ & $305(24.5 \%)$ & \\
\hline AJCC Clinical N Stage & & & $<0.001$ \\
\hline 0 & $306,097(82.2 \%)$ & 743 (59.7\%) & \\
\hline 1 & $51,235(13.8 \%)$ & $295(23.7 \%)$ & \\
\hline
\end{tabular}




\section{Cureus}

AJCC Clinical Stage Group

Histology

Ductal

ER/PR-,HER2+

\section{TABLE 2: Baseline patient and tumor characteristics by receipt of preoperative versus}

\section{postoperative radiation therapy (RT).}

preopRT: preoperative radiation therapy; postopRT: postoperative radiation therapy; IQR: Interquartile range; HSD: High school degree; AJCC: American Joint Committee on Cancer; T: primary tumor; N: regional lymph nodes; ER: Estrogen receptor; PR: Progesterone receptor; HER2: Human epidermal growth factor receptor 2.

${ }^{\text {aN }}$ ot available for patients $<40$ years old.

b Includes NCl designated comprehensive cancer centers.

${ }^{\mathrm{C}}$ Income and high school degree (HSD) are derived from patient zip code and 2012 American Community Survey data from years $2008-2012$.

dMolecular subtype was categorized into the following categories: 1) ER/PR+, HER2- (ER or PR positive and HER2 negative), 2) ER/PR+, HER2+ (ER or PR positive and HER2 positive), 3) ER/PR-, HER2+ (ER and PR negative and HER2 positive), and 4) ER/PR-, HER2- (ER, PR, and HER2 negative).

For both early-stage and locally advanced disease, higher $\mathrm{T}$ stage was associated with increased odds of preopRT use. Among early-stage patients, additional factors independently associated with preopRT use were: younger age, lower comorbidity score, higher nodal stage, and high grade (Table 3). In contrast, among patients with locally advanced disease, factors associated with preopRT were older age, less advanced nodal stage, and black race.

\begin{tabular}{|c|c|c|c|}
\hline \multirow[b]{2}{*}{ Characteristic } & \multicolumn{2}{|c|}{ Early-Stage (T1-3/N0-1) } & Locally Advanced (T4/N2-3) \\
\hline & OR $(95 \% \mathrm{Cl})$ & $\mathrm{p}$ & OR $(95 \% \mathrm{Cl})$ \\
\hline
\end{tabular}

Age, years 


\section{Cureus}

\begin{tabular}{|c|c|c|c|c|}
\hline $51-64$ & $0.93(0.79-1.10)$ & 0.381 & $1.22(0.95-1.58)$ & 0.122 \\
\hline$\geq 65$ & $0.48(0.36-0.63)$ & $<0.001$ & $2.15(1.47-3.13)$ & $<0.001$ \\
\hline White & Reference & & Reference & \\
\hline Other/Unknown & $1.13(0.84-1.52)$ & 0.406 & $1.35(0.88-2.10)$ & 0.173 \\
\hline \multicolumn{5}{|l|}{ Year of diagnosis } \\
\hline 2004-2007 & Reference & & Reference & \\
\hline 2013-2014 & $0.76(0.61-0.93)$ & 0.009 & $0.40(0.30-0.55)$ & $<0.001$ \\
\hline \multicolumn{5}{|l|}{ Charlson-Deyo Score } \\
\hline 0 & Reference & & Reference & \\
\hline$\geq 1$ & $0.78(0.63-0.98)$ & 0.035 & $0.74(0.54-1.02)$ & 0.070 \\
\hline \multicolumn{5}{|l|}{ Residence type } \\
\hline Metro & Reference & & Reference & \\
\hline \multicolumn{5}{|l|}{ Income ${ }^{a}$} \\
\hline$<\$ 38,000$ & Reference & & Reference & \\
\hline$\geq \$ 38,000$ & $0.93(0.75-1.16)$ & 0.525 & $0.87(0.65-1.16)$ & 0.351 \\
\hline \multicolumn{5}{|l|}{ Insurance Coverage } \\
\hline Uninsured & Reference & & Reference & \\
\hline Private/Managed Care & $1.07(0.66-1.74)$ & 0.790 & $0.55(0.36-0.84)$ & 0.006 \\
\hline Medicare & $1.69(0.99-2.89)$ & 0.053 & $0.51(0.30-0.85)$ & 0.010 \\
\hline Medicaid & $1.57(0.93-2.66)$ & 0.091 & $0.88(0.55-1.40)$ & 0.586 \\
\hline Unknown/Other & $1.81(0.98-3.36)$ & 0.058 & $0.82(0.39-1.72)$ & 0.594 \\
\hline \multicolumn{5}{|l|}{ AJCC Clinical T Stage } \\
\hline 1 & Reference & & Reference & \\
\hline 2 & $1.31(1.10-1.55)$ & 0.002 & $1.55(0.78-3.09)$ & 0.216 \\
\hline
\end{tabular}




\section{Cureus}

\begin{tabular}{|c|c|c|c|c|}
\hline Ductal & Reference & & Reference & \\
\hline Lobular & $0.99(0.76-1.28)$ & 0.930 & $0.47(0.27-0.82)$ & 0.008 \\
\hline Other & $1.28(0.96-1.71)$ & 0.098 & $2.05(1.45-2.90)$ & $<0.001$ \\
\hline \multicolumn{5}{|l|}{ Grade } \\
\hline Well differentiated & Reference & & Reference & \\
\hline Moderately differentiated & $1.18(0.96-1.45)$ & 0.109 & $1.00(0.61-1.64)$ & 0.989 \\
\hline Poorly differentiated & $1.69(1.36-2.09)$ & $<0.001$ & $0.98(0.60-1.59)$ & 0.925 \\
\hline Unknown & $1.70(1.25-2.30)$ & 0.001 & $1.07(0.61-1.88)$ & 0.816 \\
\hline
\end{tabular}

\section{TABLE 3: Multivariate logistic regression for the odds of receiving preoperative versus postoperative radiation therapy (RT).}

preopRT: preoperative radiation therapy; postopRT: postoperative radiation therapy; OR: Odds ratio; Cl: Confidence interval; HSD: High school degree; AJCC: American Joint Committee on Cancer; T: primary tumor; N: regional lymph nodes.

*Not applicable

Income and high school degree (HSD) are derived from patient zip code and 2012 American Community Survey data from years $2008-2012$.

In models adjusting for treatment facility type, treatment at an academic facility was associated with greater likelihood of undergoing preopRT among early-stage patients (Odds ratio [OR], 1.23; $95 \%$ confidence interval [CI], 1.05-1.43; $\mathrm{p}=0.009)$, but not for those with locally advanced disease (OR, 1.10; $95 \%$ CI, 0.87-1.38; $\mathrm{p}=$ 0.437). On multivariate analysis of patients with known molecular subtype, patients with estrogen receptor $(\mathrm{ER}) /$ progesterone receptor $(\mathrm{PR})-$, HER2- $(\mathrm{OR}, 1.30 ; 95 \% \mathrm{CI}, 1.05-1.60 ; \mathrm{p}=0.018)$ tumors were more likely to receive preopRT relative to patients with ER/PR+, HER2- tumors.

\section{Treatment characteristics}

For early-stage disease, patients treated with preopRT versus postopRT were more likely to receive neoadjuvant chemotherapy (NACT; $47.2 \%$ vs $13.4 \%$ ) and neoadjuvant hormonal therapy (NAHT; $37.4 \%$ vs $1.7 \%$; Table 4). Patients treated with preopRT versus postopRT for locally advanced disease were equally as likely to receive NACT ( $74.4 \%$ vs $73.2 \%$ ) but were more likely to receive NAHT (30.6\% vs 6.9\%). Among 695 patients who received preopRT and NACT, $95.0 \%$ received preopRT after NACT (early-stage: $96.0 \%$ vs locally advanced: $93.5 \%$; $\mathrm{p}=0.141)$. Overall, 33,716 (9.0\%) patients received no systemic therapy including 81 patients who received preopRT. Among patients who received preopRT, patients treated without systemic therapy tended to be older (median age 69 vs 56 years; $p<0.001)$ and have early-stage disease $(80.2 \%$ vs $67.4 \% ; \mathrm{p}=0.017)$. 


\section{Cureus}

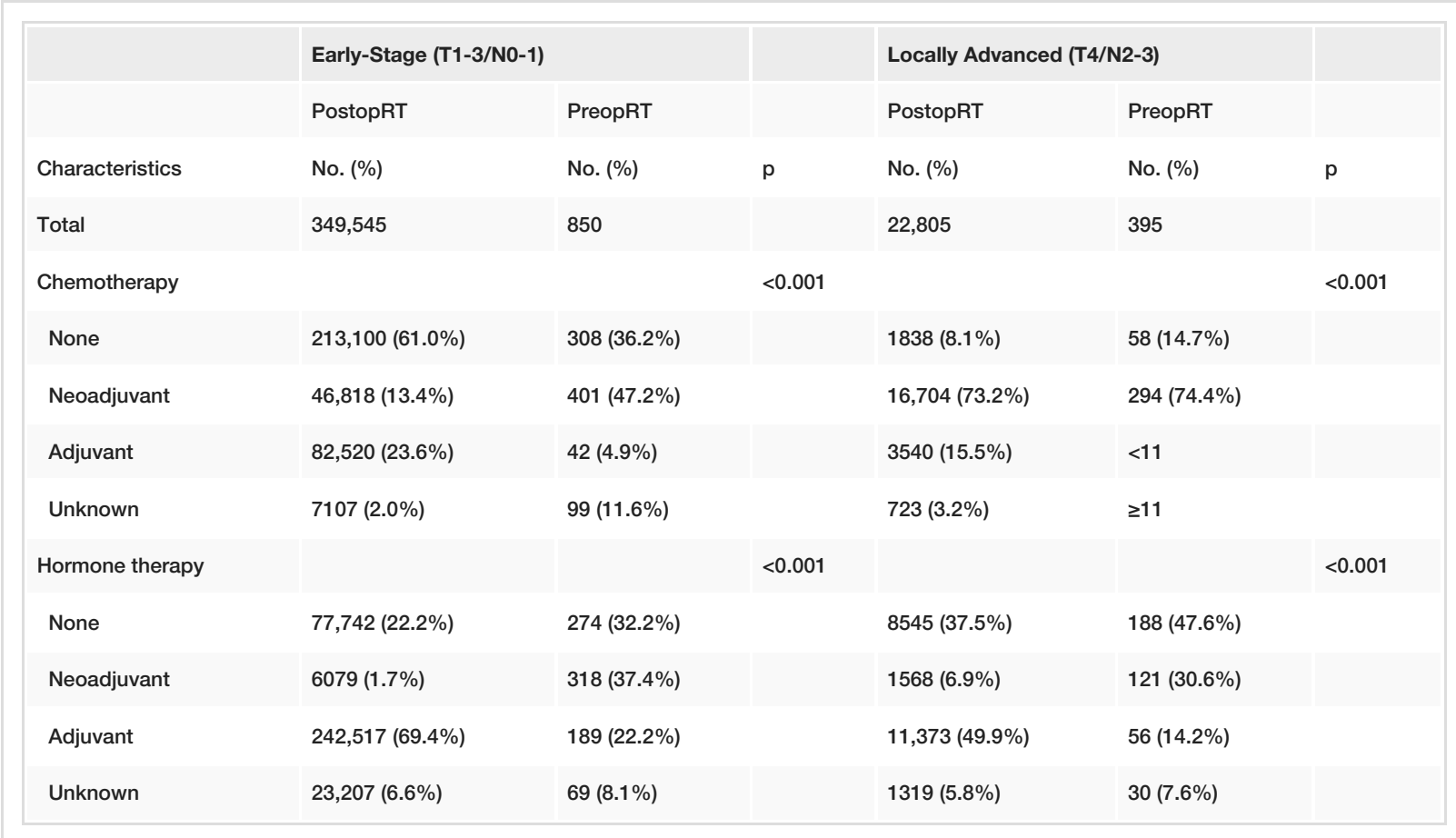

TABLE 4: Systemic therapies received within one year of definitive surgery by patients treated with preoperative versus postoperative radiation therapy (RT) in early-stage (T1-3/N0-1) and locally advanced (T4/N2-3) breast cancer.

preopRT: preoperative radiation therapy; postopRT: postoperative radiation therapy.

${ }^{*}$ In order to protect patient identity, cells with fewer than 11 patients are not shown.

Patients treated with preopRT received a median radiation dose of $59 \mathrm{~Gy}$ (interquartile range [IQR], 50-60.4 Gy) over a median of 43 days (IQR, 35-49 days). Among those who did not additionally receive postopRT (1,088 patients; $87.4 \%$ ), preopRT was completed a median of 70 days (IQR, 37-143 days) before surgery. Of patients with known radiation fields, $73.9 \%$ of early-stage patients had preopRT to the breast/chest wall only and $26.1 \%$ had preopRT to the breast/chest wall and lymph nodes. Among locally advanced patients, $40.4 \%$ had preopRT to the breast/chest wall only and $59.6 \%$ had preopRT to the breast/chest wall and lymph nodes.

\section{Surgical management and outcomes}

Patients who received preopRT were less likely to undergo breast-conserving surgery (Table 5). Compared to patients who received postopRT, patients who received preopRT were more likely to have post-mastectomy reconstruction in early-stage disease, but less likely to have reconstruction in locally advanced disease. Patients who received preopRT had a higher rate of positive surgical margins in both early-stage ( $6.0 \% \mathrm{vs}$ $4.0 \% ; \mathrm{p}=0.004)$ and locally advanced disease $(13.1 \%$ vs $7.7 \% ; \mathrm{p}<0.001)$. Rates of unplanned readmissions within 30 days of surgery were similar among patients treated with preopRT versus postopRT ( $1.2 \%$ vs $1.2 \%$; $\mathrm{p}=0.894$ ). 


\begin{tabular}{|c|c|c|c|c|c|c|c|c|c|}
\hline & \multicolumn{2}{|l|}{ Total } & & \multicolumn{2}{|c|}{ Early-Stage (T1-3/N0-1) } & & \multicolumn{2}{|c|}{$\begin{array}{l}\text { Locally Advanced } \\
\text { (T4/N2-3) }\end{array}$} & \\
\hline & \multirow{2}{*}{$\begin{array}{l}\text { PostopRT } \\
\text { n (\%) }\end{array}$} & \multirow{2}{*}{$\begin{array}{l}\text { PreopRT } \\
\text { n (\%) }\end{array}$} & \multirow{2}{*}{$\mathrm{p}$} & \multirow{2}{*}{$\begin{array}{l}\text { PostopRT } \\
\text { n (\%) }\end{array}$} & \multirow{2}{*}{$\begin{array}{l}\text { PreopRT } \\
\text { n (\%) }\end{array}$} & \multirow[b]{2}{*}{$\mathrm{p}$} & \multirow{2}{*}{$\begin{array}{l}\text { PostopRT } \\
\text { n (\%) }\end{array}$} & \multicolumn{2}{|l|}{ PreopRT } \\
\hline & & & & & & & & $\mathrm{n}(\%)$ & $\mathrm{p}$ \\
\hline $\begin{array}{l}\text { Breast conserving surgery } \\
\text { (vs mastectomy) }\end{array}$ & $\begin{array}{l}307,322 \text { of } \\
372,350 \\
(82.5 \%)\end{array}$ & $\begin{array}{l}544 \text { of } \\
1,245 \\
(43.7 \%)\end{array}$ & $<0.001$ & $\begin{array}{l}302,410 \text { of } \\
349,545 \\
(86.5 \%)\end{array}$ & $\begin{array}{l}491 \text { of } 850 \\
(57.8 \%)\end{array}$ & $<0.001$ & $\begin{array}{l}4,912 \text { of } \\
22,805 \\
(21.5 \%)\end{array}$ & $\begin{array}{l}53 \text { of } \\
395 \\
(13.4 \%)\end{array}$ & $<0.001$ \\
\hline Breast reconstruction & $\begin{array}{l}18,939 \text { of } \\
372,350 \\
(33.2 \%)\end{array}$ & $\begin{array}{l}168 \text { of } \\
1,245 \\
(28.7 \%)\end{array}$ & 0.020 & $\begin{array}{l}14,344 \text { of } \\
349,545 \\
(34.5 \%)\end{array}$ & $\begin{array}{l}131 \text { of } 850 \\
(44.1 \%)\end{array}$ & $<0.001$ & $\begin{array}{l}4,595 \text { of } \\
22,805 \\
(29.7 \%)\end{array}$ & $\begin{array}{l}37 \text { of } \\
395 \\
(12.8 \%)\end{array}$ & $<0.001$ \\
\hline $\begin{array}{l}\text { Positive (vs negative) } \\
\text { surgical margins }\end{array}$ & $\begin{array}{l}15,613 \text { of } \\
369,928(4.2 \%)\end{array}$ & $\begin{array}{l}99 \text { of } 1,213 \\
(8.2 \%)\end{array}$ & $<0.001$ & $\begin{array}{l}13,891 \text { of } \\
347,473(4.0 \%)\end{array}$ & $\begin{array}{l}50 \text { of } 839 \\
(6.0 \%)\end{array}$ & 0.004 & $\begin{array}{l}1,722 \text { of } \\
22,455 \\
(7.7 \%)\end{array}$ & $\begin{array}{l}49 \text { of } \\
374 \\
(13.1 \%)\end{array}$ & $<0.001$ \\
\hline $\begin{array}{l}\text { Readmission within } 30 \text { days } \\
\text { of surgical discharge }^{a}\end{array}$ & $\begin{array}{l}4,392 \text { of } \\
361,952(1.2 \%)\end{array}$ & $\begin{array}{l}15 \text { of } 1,201 \\
(1.2 \%)\end{array}$ & $0.894^{\#}$ & $\begin{array}{l}4,085 \text { of } \\
339,966(1.2 \%)\end{array}$ & $<11$ & $1.000^{\#}$ & $\begin{array}{l}307 \text { of } \\
21,986 \\
(1.4 \%)\end{array}$ & $<11$ & $0.661^{\#}$ \\
\hline \multicolumn{10}{|c|}{$\begin{array}{l}\text { TABLE 5: Surgical management and outcomes of patients receiving preoperative versus } \\
\text { postoperative radiation therapy (RT) in early-stage (T1-3/N0-1) and locally advanced (T4/N2-3) } \\
\text { breast cancer. }\end{array}$} \\
\hline \multicolumn{10}{|c|}{ preopRT: preoperative radiation therapy; postopRT: postoperative radiation therapy. } \\
\hline \multicolumn{10}{|c|}{ *In order to protect patient identity, cells with fewer than $11 \mathrm{pa}$} \\
\hline \multicolumn{10}{|l|}{ \#Fisher's exact test } \\
\hline iplanned readmission to the & treating hospital u & ithin 30 days $o$ & f discharg & ge following hospit & italization for $\mathrm{I}$ & resection & of the pri & y cance & \\
\hline
\end{tabular}

In total, $14.8 \%$ of preopRT patients had response to neoadjuvant therapy recorded in the database with an overall pathologic complete response (pCR) rate of $25.5 \%$. Of the 90 early-stage and 94 locally advanced patients who had preopRT with recorded pCR, pCR rates were $32.2 \%$ and $19.1 \%$, respectively. Among 83 patients who had preopRT, ER/PR+, HER2- tumors, and pCR recorded in the database, pCR rate was $18.1 \%$. Of the 46 early-stage and 37 locally advanced patients who had preopRT and ER/PR+, HER2- tumors with recorded $\mathrm{pCR}$, pCR rates were $23.9 \%$ and $10.8 \%$, respectively.

\section{Discussion}

The traditional paradigm of surgery followed by possible RT and/or chemotherapy for patients with breast cancer has been challenged by the increased use of neoadjuvant systemic therapy and interest in preopRT [8]. Our analysis evaluating patterns of care with preopRT in the U.S. through a large hospital-based registry of breast cancer patients contributes to the growing literature on preopRT in breast cancer, which up until now has been primarily comprised of single institution experiences. We characterize several distinct patient populations that have received preopRT thereby furthering our understanding of its potential applications.

We found that use of preopRT varied by demographic and tumor characteristics, suggesting there may be different indications for its use across treatment settings. Patients undergoing preopRT were more likely to be younger, black, and uninsured or Medicaid-insured. Furthermore, these patients were more likely to present with T3-4 tumors, more advanced nodal disease, and triple negative subtype. These findings build upon previously published literature highlighting socioeconomic disparities among breast cancer patients [9, 10]; patients of lower socioeconomic status were likely selected for neoadjuvant treatment with preopRT due to presentation with more advanced disease. Younger age and nodal involvement (N1 vs N0) predicted for use of preopRT in the setting of early-stage disease, but older age and N0 disease predicted for use of preopRT in patients with locally advanced disease. It may be that younger women with early-stage disease and positive nodes undergo preopRT to de-escalate surgery of the breast and axilla similar to the emerging role of NACT in this setting [11]. PreopRT may be an alternative to NACT or an adjunct to NAHT in older, locally advanced patients with negative nodes, since endocrine therapy is associated with slow, partial tumor responses [12]. PreopRT may also be used in patients demonstrating less of a response to NACT, as we found 
95\% of patients who received NACT and preopRT underwent preopRT after NACT [8]. Finally, we found that treatment at an academic facility was associated with use of preopRT in early-stage disease, but not locally advanced disease, which highlights the novelty of this approach in early-stage breast cancer.

Given that women who undergo breast conservation have reported higher satisfaction with body image than women who undergo mastectomy, a neoadjuvant strategy that facilitates breast-conserving treatment is important [13]. However, our analysis does not inform us regarding the reasons preopRT was chosen among patients. The significantly lower rates of breast conservation, $57.8 \%$ vs $86.5 \%$, for the early-stage preopRT group may be due to presentation with relatively more advanced disease, sociodemographic drivers of treatment decisions, patient preferences, and surgeon recommendations [14]. Other factors that are relevant when discussing breast conservation include whether neoadjuvant treatment can improve rates of marginnegative resection and pCR. In the NACT setting, no standard approach to breast conservation and margin interpretation has been delineated. There are conflicting reports about whether NACT is associated with lower [2, 15] or higher [16] rates of re-excision following lumpectomy. We found that preopRT was associated with higher rates of positive surgical margins (8.2\% vs $4.2 \%)$. These results may be attributed to selection bias, as preopRT may have been motivated by poor response to NACT or NAHT, lesion location in the breast, or size of the lesion with respect to the breast. However, a heterogeneous tumor response with mosaic patterns of tumor shrinkage could have contributed to greater uncertainty during surgical resection. Additionally, depending on the timing of preopRT with respect to operative intervention, the degree of tissue inflammation or induration encountered could have further impaired surgical excision. Further work is needed to establish the optimal timing of surgery after preopRT $[1,6,7,17]$.

In our analysis, $25 \%$ of patients receiving preopRT achieved pCR (32\% early-stage and 19\% locally advanced). Among patients with ER/PR+, HER2- tumors, 18\% of patients receiving preopRT achieved pCR (24\% earlystage and $11 \%$ locally advanced). In contrast, among patients with low-grade, hormone receptor-positive tumors, the rate of pCR following NACT and/or NAHT alone is low, ranging from 2 to $10 \%$ [18, 19]. Furthermore, pCR is not a strong prognostic marker among these patients [20]. Although our pCR analysis was limited by the low number of patients with this data available, these favorable pCR rates raise the intriguing possibility of the potential role of preopRT to increase pCR and as a tool of bioselection analogous to the emerging role that NACT plays in patients with hormone receptor-negative disease.

Other studies of preopRT have reported lower rates of pCR. Nichols et al. conducted a prospective trial of preoperative accelerated partial breast irradiation in patients with early-stage breast cancer and found a pCR rate of $15 \%$ among patients with ER+ tumors [6]. However, no patients on this trial received NACT or NAHT. In the locally advanced setting, Adams et al. showed that pCR was associated with significantly better disease-free and overall survival following preoperative concurrent paclitaxel-radiation [21].

In addition to improving rates of breast-conserving surgery, there is interest in using preopRT to facilitate immediate breast reconstruction. PreopRT followed by combined oncologic and reconstructive surgery may be preferable to traditional sequencing with postopRT because it may reduce the shrinkage of transferred tissue and affect perfusion or contracture around implants [8]. We found higher rates of reconstruction among early-stage preopRT patients and lower rates of reconstruction among locally advanced preopRT patients; however, these rates may be driven by sociodemographic rather than tumor characteristics [22-24].

Lastly, we found that preopRT was not associated with increased risk of readmission within 30 days of surgical discharge. This finding is corroborated by multiple studies demonstrating acceptable postoperative complication rates among breast cancer patients receiving preopRT prior to mastectomy with or without immediate breast reconstruction [25-27].

To our knowledge, this is the first nationwide study to detail the use of preopRT in newly diagnosed breast cancer patients. The strengths of this analysis are that it encompasses a contemporary population from a hospital-based registry reflective of the U.S. population. Our conclusions are confounded by indication, missing/unknown data such as molecular subtype, lack of pCR coding in the NCDB for greater than $90 \%$ of patients, and no specification regarding the use of partial breast irradiation. We also could not account for unmeasured confounders such as genetic testing, family history, patient preferences, and provider recommendations. Our assessment of complications is limited to 30-day surgical readmissions and does not account for late complications or less severe complications not requiring hospital admission. Lastly, our results may only apply to women treated at hospitals accredited by the Commission on Cancer and the American Cancer Society, which are enriched for hospitals with strong oncology services [28].

\section{Conclusions}

The role of preopRT is being reconsidered for patients with breast cancer with different clinical and sociodemographic drivers of its use in the early-stage and locally advanced settings. This approach may improve rates of pCR in hormone receptor-positive tumors and facilitate breast reconstruction in earlystage disease. We await the results of ongoing trials that will shed further light on the feasibility and effectiveness of preopRT. 


\section{Additional Information \\ Disclosures}

Human subjects: Consent was obtained by all participants in this study. Exempt from review issued approval N/A. Animal subjects: All authors have confirmed that this study did not involve animal subjects or tissue. Conflicts of interest: In compliance with the ICMJE uniform disclosure form, all authors declare the following: Payment/services info: All authors have declared that no financial support was received from any organization for the submitted work. Financial relationships: All authors have declared that they have no financial relationships at present or within the previous three years with any organizations that might have an interest in the submitted work. Other relationships: All authors have declared that there are no other relationships or activities that could appear to have influenced the submitted work.

\section{Acknowledgements}

Preliminary results of this work were presented at the 2018 Annual Meeting of the American Society for Radiation Oncology (ASTRO) held October 21-24 in San Antonio, Texas.

\section{References}

1. Horton JK, Blitzblau RC, Yoo S, et al.: Preoperative single-fraction partial breast radiation therapy: a novel phase 1, dose-escalation protocol with radiation response biomarkers. Int J Radiat Oncol Biol Phys. 2015, 92:846-855. 10.1016/j.ijrobp.2015.03.007

2. Christy CJ, Thorsteinsson D, Grube BJ, et al.: Preoperative chemotherapy decreases the need for re-excision of breast cancers between 2 and 4 cm diameter. Ann Surg Oncol. 2009, 16:697-702. 10.1245/s10434-0080268-5

3. Wennerberg E, Lhuillier C, Vanpouille-Box C, et al.: Barriers to radiation-induced in situ tumor vaccination . Front Immunol. 2017, 8:229. 10.3389/fimmu.2017.00229

4. Vatner RE, Cooper BT, Vanpouille-Box C, Demaria S, Formenti SC: Combinations of immunotherapy and radiation in cancer therapy. Front Oncol. 2014, 4:325. 10.3389/fonc.2014.00325

5. van der Leij F, Bosma SCJ, van de Vijver MJ, et al.: First results of the preoperative accelerated partial breast irradiation (PAPBI) trial. Radiother Oncol. 2015, 114:322-327. 10.1016/j.radonc.2015.02.002

6. Nichols E, Kesmodel SB, Bellavance E, et al.: Preoperative accelerated partial breast irradiation for earlystage breast cancer: preliminary results of a prospective, phase 2 trial. Int J Radiat Oncol Biol Phys. 2017, 97:747-753. 10.1016/j.ijrobp.2016.11.030

7. Bollet MA, Belin L, Reyal F, et al.: Preoperative radio-chemotherapy in early breast cancer patients: longterm results of a phase II trial. Radiother Oncol. 2012, 102:82-88. 10.1016/j.radonc.2011.08.017

8. Lightowlers SV, Boersma LJ, Fourquet A, et al.: Preoperative breast radiation therapy: Indications and perspectives. Eur J Cancer. 2017, 82:184-192. 10.1016/j.ejca.2017.06.014

9. Bradley CJ, Given CW, Roberts C: Race, socioeconomic status, and breast cancer treatment and survival . J Natl Cancer Inst. 2002, 94:490-496. 10.1093/jnci/94.7.490

10. Dreyer MS, Nattinger AB, McGinley EL, Pezzin LE: Socioeconomic status and breast cancer treatment . Breast Cancer Res Treat. 2018, 167:1-8. 10.1007/s10549-017-4490-3

11. King TA, Morrow M: Surgical issues in patients with breast cancer receiving neoadjuvant chemotherapy . Nat Rev Clin Oncol. 2015, 12:335-343. 10.1038/nrclinonc.2015.63

12. Ellis MJ, Babiera G, Unzeitig GW, et al.: ACOSOG Z1031: A randomized phase II trial comparing exemestane, letrozole, and anastrozole in postmenopausal women with clinical stage II/III estrogen receptor-positive breast cancer. J Clin Oncol. 2010, 28:513. 10.1200/jco.2010.28.18_suppl.lba513

13. Engel J, Kerr J, Schlesinger-Raab A, Sauer H, Hölzel D: Quality of life following breast-conserving therapy or mastectomy: results of a 5-year prospective study. Breast J. 2004, 10:223-231. 10.1111/j.1075122X.2004.21323.X

14. Gu J, Groot G, Boden C, Busch A, Holtslander L, Lim H: Review of factors influencing women's choice of mastectomy versus breast conserving therapy in early stage breast cancer: a systematic review. Clin Breast Cancer. 2018, 18:e539-e554. 10.1016/j.clbc.2017.12.013

15. Waljee JF, Hu ES, Newman LA, Alderman AK: Predictors of re-excision among women undergoing breastconserving surgery for cancer. Ann Surg Oncol. 2008, 15:1297. 10.1245/s10434-007-9777-x

16. Song JH, Park JY, Choi JE, Kang S, Lee SJ, Bae Y: Re-excision rate in breast conservation surgery after neoadjuvant chemotherapy. J Breast Dis. 2017, 5:16-22. 10.14449/jbd.2017.5.1.16

17. Brackstone M, Palma D, Tuck AB, et al.: Concurrent neoadjuvant chemotherapy and radiation therapy in locally advanced breast cancer. Int J Radiat Oncol Biol Phys. 2017, 99:769-776. 10.1016/j.ijrobp.2017.06.005

18. Colleoni M, Montagna E: Neoadjuvant therapy for ER-positive breast cancers . Ann Oncol. 2012, 23:243-248. 10.1093/annonc/mds305

19. Spring LM, Gupta A, Reynolds KL, et al.: Neoadjuvant endocrine therapy for estrogen receptor-positive breast cancer: a systematic review and meta-analysis. JAMA Oncol. 2016, 2:1477-1486. 10.1001/jamaoncol.2016.1897

20. Cortazar P, Zhang L, Untch M, et al.: Pathological complete response and long-term clinical benefit in breast cancer: the CTNeoBC pooled analysis. Lancet. 2014, 384:164-172. 10.1016/s0140-6736(13)62422-8

21. Adams S, Chakravarthy AB, Donach M, et al.: Preoperative concurrent paclitaxel-radiation in locally advanced breast cancer: pathologic response correlates with five-year overall survival. Breast Cancer Res Treat. 2010, 124:723-732. 10.1007/s10549-010-1181-8

22. Jagsi R, Jiang J, Momoh AO, et al.: Trends and variation in use of breast reconstruction in patients with breast cancer undergoing mastectomy in the United States. J Clin Oncol. 2014, 32:919-926.

10.1200/JCO.2013.52.2284

23. August DA, Wilkins E, Rea T: Breast reconstruction in older women. Surgery. 1994, 115:663-668. 


\section{Cureus}

24. Newman LA, Kuerer HM, Hunt KK, et al.: Feasibility of immediate breast reconstruction for locally advanced breast cancer. Ann Surg Oncol. 1999, 6:671-675. 10.1007/s10434-999-0671-6

25. Riet FG, Fayard F, Arriagada R, et al.: Preoperative radiotherapy in breast cancer patients: 32 years of follow-up. Eur J Cancer. 2017, 76:45-51. 10.1016/j.ejca.2017.01.022

26. Paillocher N, Florczak AS, Richard M, et al.: Evaluation of mastectomy with immediate autologous latissimus dorsi breast reconstruction following neoadjuvant chemotherapy and radiation therapy: a single institution study of 111 cases of invasive breast carcinoma. Eur J Surg Oncol. 2016, 42:949-955.

10.1016/j.ejso.2016.03.024

27. Baltodano PA, Reinhardt ME, Flores JM, et al.: Preoperative radiotherapy is not associated with increased post-mastectomy short-term morbidity: analysis of 77,902 patients. Plast Reconstr Surg Glob Open. 2017, 5:1108. 10.1097/GOX.0000000000001108

28. Bilimoria KY, Stewart AK, Winchester DP, Ko CY: The National Cancer Data Base: a powerful initiative to improve cancer care in the United States. Ann Surg Oncol. 2008, 15:683-690. 10.1245/s10434-007-9747-3 